\title{
Therapeutic strategies in primary malignancies of the vagina - a literature review
}

\author{
Nicolae BACALBASA ${ }^{1}$, Olivia IONESCU ${ }^{2}$, Irina BALESCU ${ }^{3}$ \\ 1"Carol Davila" University of Medicine and Pharmacy, Bucharest \\ 2"Bucur" Maternity Hospital, Bucharest \\ 3"Ponderas" Hospital, Bucharest
}

\begin{abstract}
Primary vaginal cancers are very rare malignancies accounting for only 1 to $2 \%$ of all gynecologic malignancies. The most frequent histologic subtype is squamous cell carcinoma followed by adenocarcinoma. Due to its rarity, data concerning the natural history, prognostic factors, and treatment are rather poor; however an extension of the reported clinical experience for cervical and anal cancer has been proposed given the similarities in disease etiology and the desire for organ preservation. This is a literature review of the most appropriate therapeutic strategies used in vaginal cancer management.
\end{abstract}

Keywords: vaginal cancer, squamous cell carcinoma, radiation therapy

Abbreviations

SCC = squamous cell carcinoma; VAIN = vaginal intraepithelial neoplasia;

EBRT = external beam radiation therapy; $\mathbf{R T}$ = radiotherapy

\section{INTRODUCTION}

The overall reported incidence of vaginal cancer is 0.45 cases per 100000 women with lower rates in white (0.42) compared with black (0.73) and Hispanic women (0.56) (1). The median age at diagnosis is 58 . According to FIGO classification, tumors should be classified as carcinoma of the vagina only when the primary place of growth is the vagina therefore being necessary the exclusion of cervical, vulvar or urethral origins (2).

When talking about the most common seen histopathological subtypes, squamous cell carcinoma (SCC) represents more than $90 \%$ of all primary tumors while adenocarcinomas account for approximately $5 \%$ (3). Other histological subtypes are very rare and include malignant melanomas, neuroendocrine carcinomas and papillary squamo-transitional cell carcinomas 
(4). Müllerian adenosarcoma developing from vaginal endometriosis or endometrioid carcinoma have also been described in the literature but their incidence remains very low $(5,6)$.

Due to the low incidence of the primary vaginal tumors there is a wide variety of therapeutic modalities to which women can be subjected to. This review focuses on the most appropriate treatment options for the primary vaginal cancer.

\section{RISK AND PROGNOSTIC FACTORS}

The peak incidence of SCC is reached during the sixth and seventh decades while less than $15 \%$ of patients are diagnosed below the age of 50 years, and $<10 \%$ of these tumors occur in patients under the age of 40 years. SCC vaginal cancer frequently metastasizes to the lung and liver (4).

SCC of the vagina has been linked with the human papillomavirus infection, specifically with serotypes 16 and 18 which were identified in more than $50 \%$ of all patients. Due to the association with HPV infection, vaginal in situ and invasive SCC present similar risk factors to the cervical cancer. Vaginal intraepithelial neoplasia (VAIN) is a precursor to invasive cervical cancer and almost $2 \%$ of patients with VAIN will progress to invasive cancer (7).

Adenocarcinomas usually occur in younger patients, with ages ranging between 17 and 21 years; the sites of distant metastases are lung, supraclavicular and pelvic nodes (5). Clear cell adenocarcinomas usually occur in women below the age of 30 years who had been exposed to synthetic estrogen-diethylstilbestrol (8).

The prognosis of primary vaginal tumors seems to be strongly correlated with FIGO stage at the moment of diagnosis; it seems that the initial FIGO stage significantly impacts on the rate of survival also being correlated to the development of local recurrence or distant metastasis (9). Other incriminated prognostic factors are the tumor size and location and patient's age, but their impact on the prognosis is still a matter of debate (10).

Several studies have reported that cancers developing in the proximal half of the vagina have a better survival and a decreased recurrence rate when compared to lesions involving the distal half or the entire length of the vagina. Lesions of the posterior vaginal wall have a worse prognosis with a higher incidence of lymph node metastasis than cancers located in the anterior wall or the vaginal apex (11-13).

Another prognostic factor is the lymph nodal status. The lymphatic flow from the upper third of the vagina drains primarily at the level of common, internal and external iliac lymph nodes while the lower part of the vagina drains into the inguino-femoral lymph nodes (14). The histologic type and grade are independent predictors of survival, a higher incidence of local recurrences being observed in women with adenocarcinoma than those with SCC (11).

\section{STAGING AND MANAGEMENT}

According to FIGO staging of the lesion, various imagistic studies have been proposed including chest radiography, cystoscopy, rectoscopy and intravenous urography; CT scanning, MR imaging and FDG-PET may also help to guide therapy (15).

The staging system for vaginal cancer is rather based on clinical criteria.

Stage I disease is limited to the vaginal wall, stage II disease involves the surrounding tissues with no extent to the pelvic wall, stage III tumor extends to the pelvic wall while stage IV tumors extend beyond the true pelvis or present local invasion of the bladder or rectal mucosa. The therapeutic options depend on FIGO stage of the tumor, lesion's location and histopathological subtype. Most data available in the literature concerning the surgical and radio-therapeutic procedures refer to SCC of the vagina (16).

\section{Stage I}

The treatment approaches for stage I include RT with or without surgery. In stage I vaginal cancer, the rate of lymph node involvement ranges from 6 to 16\% (17). Surgery in this stage refers to: wide local excision with disease with tumor free margins (for small lesions) or more extended resections such as partial vaginectomy, total simple vaginectomy and radical vaginectomy (removal of the involved vagina and paravaginal tissue to the pelvic sidewall) for larger lesions. Vulvovaginectomy and dissection of the inguinofemoral lymphatic nodes seems to be the most appropriate approach for cancers located in the lower third of the vagina followed by adjuvant RT if the margins are positive for tumor invasion after resection (17).

Reports from the National Cancer Database show that in women with FIGO stage I of the disease surgery alone has clinically and statistically 
better results than $\mathrm{RT}$ with a 5 -year relative survival rate of $56-90 \%$ for cases treated only with surgery; however these rates significantly improve whether adjuvant radiation therapy is associated (with a reported rate of 5 year overall survival of $79-100 \%$ ). Studies have shown that this improvement has been particularly obtained in cases at risk to develop local recurrences $(16,18)$.

RT alone with the use of interstitial (singleplan implant) and intracavitary therapy with a minimum dose of $75 \mathrm{~Gy}$ is an accepted method of treatment for stage I vaginal cancer with a cumulative 5-year survival rate between $33-100 \%$ $(11,19)$. Pelvic failure rates following brachytherapy alone range from $14 \%$ to $33 \%$. Anterior, posterior, or total exenteration is performed less commonly for early-stage disease, as radiation therapy is the preferred modality for organ preservation $(10,11)$.

External Beam Radiotherapy (EBRT) can be used for large, infiltrative or poorly differentiated tumors which are associated with a high risk of lymph node metastasis or loco-regional failure (20).

\section{Stage II}

The standard treatment of this stage consists in brachytherapy associated with EBRT, a therapeutic strategy which has been associated with improved pelvic control and improved rates of survival $(21,22)$. The predominant pattern of failure following RT is locoregional, similar to the one encountered after surgery (22).

Interstitial brachytherapy has been proved to have better results in terms of disease free survival and local control when compared to intracavitary therapy (22). The choice between intracavitary or interstitial radiotherapy depends on the site and size of the tumor. Intracavitary radiation therapy is used if the residual disease is no more than 3-5 mm from the vaginal surface. Patients with deeper lesions are treated with interstitial implants. Tumors of the middle third of the vagina are handled with intracavitary and interstitial brachytherapy. Interstitial radiation therapy boosts are used for the tumors of the distal part of the vagina; in these cases pelvic and inguinal irradiation might be taken in consideration if lymph node metastases at these levels are expected $(23,24)$.

There are few studies which examine the impact of RT combined with systemic cytotoxic agents such as 5-Fluoro-Uracil or Mitomycin-C on the local control and survival in patients with stage II vaginal cancer. Dalrymple et al conducted a study involving 14 cases diagnosed with stages I and II vaginal cancer and reported a 5 -year overall survival of $93 \%$ after a protocol which included the association of RT (with doses of $63 \mathrm{~Gy}$ ) and chemotherapeutic regimens based on 5-Fluoro-Uracil (25).

Further investigation is required to determine if women with stage II disease can be cured with radical surgery. Total vaginectomy or an exenterative procedure is required in order to obtain negative surgical margins (14). Laparoscopic radical vaginectomy with the construction of a neovagina has been proposed as an organ-sparing surgical alternative (26).

Neoadjuvantchemotherapy (NACT) with cisplatin and paclitaxel followed by radical vaginectomy and pelvic lymphadenectomy might provide a partial and respectively a complete response in $64 \%$ and respectively $27 \%$ of cases (27). In patients with an incomplete resection or positive lymph nodes, adjuvant RT might provide a 5 -year survival rate of $40-69 \%(9,14,17)$.

\section{Treatment of locally advanced disease (FIGO stages III-IV)}

The association between EBRT and brachytherapy remains the treatment of choice for stage III vaginal cancer $(22,28)$. EBRT administered for 6 weeks appears to improve survival (22) while brachytherapy is important for the local management of the disease (28). In selected cases another appropriate approach is pelvic exenteration or a combination between irradiation and exenteration $(9,18)$.

For stage IVA of the disease the most appropriate therapeutic approach consists in a combination between interstitial, intracavitary RT and EBRT with reported 5 year survival rates of $0-62 \%(9,18,22,28)$. For women with stage IV A who are in good general condition a pelvic exenteration with vaginal reconstruction using a gracilis myocutaneous flap or rectus abdominis myocutaneous flap may be a treatment option with a 5 -year survival rate of $0-50 \%$; a similar approach might also be useful in cases necessitating palliation in order to treat a locally advanced vaginal cancer associated with rectovaginal or vesicovaginal fistula $(16,29)$.

For stage IV B of the disease, palliative radiation therapy with or without simultaneous chemotherapy remains the most appropriate therapeutic option $(11,18)$.

The cause-specific survival rates of the advanced-stage vaginal cancer range from $23-59 \%$ 
for stage III of the disease and from 0-25\% for stage IV of the disease. Corresponding pelvic control rates are $62-71 \%$ and respectively $12-30 \%(30,31)$. However, almost $80 \%$ of patients report persistent or recurrent pelvic confined disease after EBRT or brachytherapy. When talking about distant metastases, their incidence reaches almost $25-30 \%$ among patients with locally advanced tumors (32).

A survival advantage for the use of NACT prior to radical surgery has not been found in randomized trials of patients with locally advanced vaginal cancer; however experience in this direction proved to be limited $(32,33)$.

\section{Concurrent chemoradiotherapy}

Although there are no prospective trials for the use of concurrent RT in patients with vaginal cancer, institutional reports support the utility of chemoradiotherapy based on survival rates observed in trials of locally advanced cervival cancer (34). Series of patients with early- and late-stage of the disease had a local control rate of $92 \%$ and a 5 -year progression-free survival rate of $75 \%$ with concurrent weekly administration of cisplatin $\left(40 \mathrm{mg} / \mathrm{m}^{2}\right)$. A series of 26 patients with predominately locally advanced disease was treated with definitive RT and 5-FU with or without mitomycin $C$ or weekly singleagent cisplatin. A 5 -year survival rate of $50 \%$ and a pelvic failure of $31 \%$ were reported (35).

\section{Treatment considerations and follow-up}

Treatment-related factors associated with poor survival in vaginal cancer are prolonged overall treatment time and low hemoglobin levels. RT should be completed within 8-9 weeks and transfusion should be performed in order to maintain hemoglobin levels of $>10-11 \mathrm{~g} / \mathrm{dl}$. Prolongation of the treatment time does not appear to have a significant impact on pelvic tumor control (31). Some authors emphasized the potential advantage in terms of disease-free survival and local control using interstitial RT when compared to the use of intracavitary therapy $(14,22)$.
The follow-up for vaginal cancer implies a clinical examination every 3 months for 2 years followed by less frequent intervals after 2 years. Based on the practice for locally advanced cervical cancer, consideration of post-treatment PET/ CT surveillance is reasonable for patients with initial bulky disease (36).

\section{CONCLUSIONS}

Vaginal cancer is a very rare disease which is frequently diagnosed when the disease has already involved the submucosal layer and sometimes the pelvic sidewall. Moreover, the prevalence of the disease in elderly patients makes the treatment more difficult. The management depends on the extent of disease, the presence of comorbidities, and the desire to maintain the ovarian and/or sexual function.

For cases diagnosed in FIGO stage I, the most appropriate treatment consists in radical surgery with or without adjuvant radiotherapy. The studies carried out on retrospective series have shown equivalence or even the superiority of surgery compared to RT alone in FIFO stage I. In FIGO stage II the standard treatment remains the combination of brachytherapy and EBRT or radical surgery for selected patients. NACT followed by radical surgery is a valid alternative to the standard treatment in terms of survival.

Combination of EBRT and brachytherapy followed by resection is the most efficient option in FIGO stages III-IV A. For women with FIGO stage IVB of the disease palliative RT may play an important role in alleviating the pain and bleeding associated with uncontrolled pelvic disease.

Further data are required for the use of concurrent chemo-radiotherapy as a treatment strategy.

However, it seems that the management strategies introduced in cervical cancer, such as $\mathrm{NACT}+\mathrm{RT}$ will be applicable to primary vaginal cancer too; similar survival rates are expected, without affecting the quality of life. 


\section{REFERENCES}

1. Siegel R., Naishadham D., Jemal A. Cancer statistics, 2013. CA Cancer J Clin. 2013; 63:11-30

2. Creasman W.T., Phillips J.L., Menck H.R. The National Cancer Data Base report on cancer of the vagina. Cancer. 1998; 83:1033-40

3. Lilic V., Lilic G., Filipovic S., Visnjic M., Zivadinovic R. Primary carcinoma of the vagina. J BUON. 2010 Apr-Jun; 15(2):241-7

4. Hacker N.F., Eifel P.J., van der Velden J. Cancer of the vagina. Int $J$ Gynaecol Obstet. 2012; 119(Suppl. 2):S97-9

5. Nomoto K., Hori T., Kiya C., et al. Endometrioid adenocarcinoma of the vagina with a microglandular pattern arising from endometriosis after hysterectomy. Pathol Int. 2010; 60:636-41

6. Liu L., Davidson S., Singh M. Müllerian adenosarcoma of vagina arising in persistent endometriosis: report of a case and review of the literature. Gynecol Oncol. 2003; 90:486-9

7. Daling J.R., Madeleine M.M., Schwartz S.M., et al. A population-based study of squamous cell vaginal cancer: HPV and cofactors. Gynecol Oncol. 2002; 84:263-70

8. Renaud M.C., Plante M., Grégoire J., Roy M. Primitive clear cell carcinoma of the vagina treated conservatively. J Obstet Gynaecol Can. 2009; 31:54-6

9. Rubin S.C., Young J., Mikuta J.J. Squamous carcinoma of the vagina: treatment, complications and longterm follow-up. Gynecol Oncol. 1985; 20:346-53

10. Beller U., Bnedet J.L., Creasman W.T., et al. Carcinoma of the vagina. FIGO 6th Annual Report on the Results of Treatment in Gynecological Cancer. Int J Gynaecol Obstet. 2006; 95(November (Suppl. 1)):S29-42

11. Kucera H., Vavra N. Radiation management of primary carcinoma of the vagina: clinical and histopathological variables associated with survival. Gynecol Oncol. 1991; 40:12-6

12. Urbanski K., Kojs Z., Reinfuss M., et al. Primary invasive vaginal carcinoma treated with radiotherapy: analysis of prognostic factors. Gynecol Oncol. 1996; 60:16-21

13. Tarraza Jr M.H., Muntz H., DeCain M., et al. Patterns of recurrence of primary carcinoma of the vagina. Eur $\mathrm{J}$ Gynecol Oncol. 1991; 12: 89-92
14. Stock R.G., Chen A.S.J., Seski J. A 30-year experience in the management of primary carcinoma of the vagina: analysis of prognostic factors and treatment modalities. Gynecol Oncol. 1995;56:45-52

15. Current FIGO staging for cancer of the vagina, fallopian tube, ovary, and gestational trophoblastic neoplasia. Int J Gynaecol Obstet. 2009;105:3-4

16. Ball H.G., Berman M.L. Management of primary vaginal carcinoma. Gynecol Oncol. 1982; 14:154-63

17. Davis K.P., Stanhope C.R., Garton G.R., et al. Invasive vaginal carcinoma: analysis of early stage disease. Gynecol Oncol 1991; 42:131-6

18. Siegel R., Naishadham D., Jemal A. Cancer statistics, 2013. CA Cancer J Clin. 2013; 63:11-30

19. Tran P.T., Su Z., Lee P., et al. Prognostic factors for outcomes and complications for primary squamous cell carcinoma of the vagina treated with radiation. Gynecol Oncol. 2007; 105:641-9

20. Mock U., Kucera H., Fellner C., et al. High-dose-rate (HDR) brachytherapy with or without external beam radiotherapy in the treatment of primary vaginal carcinoma: long-term results and side effects. Int $\mathrm{J}$ Radiat Oncol Biol Phys. 2003; 56:950-7

21. Chyle V., Zagars G.K., Wheeler J.A., et al. Definitive radiotherapy for carcinoma of the vagina. Int J Radiat Oncol Biol Phys. 1996; 35:891-905

22. Perez C.A., Grigsby P.W., Garipagaoglu M., et al. Factors affecting longterm outcome of irradiation in carcinoma of the vagina. Int J Radiat Oncol Biol Phys. 1999; 44:37-45

23. Chu A.M., Beechinor R. Survival and recurrence patterns in the radiation treatment of carcinoma of the vagina. Gynecol Oncol. 1984; 19:298-307

24. Lee W.R., Marcus Jr R.B., Sombeck M.D., et al. Radiotherapy alone for carcinoma of the vagina: the importance of overall treatment time. Int J Radiat Oncol Biol Phys. 1994; 29:983

25. Dalrymple J.L., Russell A.H., Lee S.W., et al. Chemoradiation for primary invasive squamous carcinoma of the vagina. Int $\mathrm{J}$ Gynaecol Cancer. 2004; 14:110-7

26. Ling B., Gao Z., Sun M., et al. Laparoscopic radical hysterectomy with vaginectomy and reconstruction of vagina in patients with stage I of primary vaginal carcinoma. Gynecol Oncol. 2008;109:92-6

27. Benedetti Panici P., Bellati F., Plotti F., et al. Neoadjuvant chemotherapy followed by radical surgery in patients affected by vaginal carcinoma. Gynecol Oncol. 2008; 111(2):307-11

28. Tewari K.S., Cappuccini F., Puthawala A.A., et al. Primary invasive carcinoma of the vagina: treatment with interstitial brachytherapy. Cancer. 2001; 91:758-70

29. Reddy S., Saxena V.S., Reddy S., et al. Results of radiotherapeutic management of primary carcinoma of the vagina. Int $\mathrm{J}$ Radiat Oncol Biol Phys. 1991; 21(September (4)):1041-4

30. Crevoisier R., Sanfilippo N., Gerbaulet A., et al. Exclusive radiotherapy for primary squamous cell carcinoma of the vagina. Radiother Oncol. 2007; 85:362-70

31. Tran P.T., Su Z., Lee P., et al. Prognostic factors for outcomes and complications for primary squamous cell carcinoma of the vagina treated with radiation. Gynecol Oncol. 2007; 105:641-9

32. Frank S.J., Jhingran A., Levenback C., Eifel P.J. Definitive radiation therapy for squamous cell carcinoma of the vagina. Int J Radiat Oncol Biol Phys. 2005; 62:138-47

33. Rydzewska L., Tierney J., Vale C.L., Symonds P.R. Neoadjuvant chemotherapy plus surgery versus surgery for cervical cancer. Cochrane Database Syst Rev. 2010; CD007406

34. Lanciano R., Calkins A., Bundy B.N., et al. Randomized comparison of weekly cisplatin or protracted venous infusion of fluorouracil in combination with pelvic radiation in advanced cervix cancer: a gynecologic oncology group study. J Clin Oncol. 2005; 23:8289-95

35. Samant R., Lau B., E C., et al. Primary vaginal cancer treated with concurrent chemoradiation using Cis-platinum. Int J Radiat Oncol Biol Phys. 2007; 69:746-50

36. Dimopoulos J.C., Schmid M.P., Fidarova E., et al. Treatment of locally advanced vaginal cancer with radiochemotherapy and magnetic resonance image-guided adaptive brachytherapy: dose-volume parameters and first clinical results. Int $J$ Radiat Oncol Biol Phys. 2012; 82:1880-8 